\title{
Towards a Virtual Research Environment for Paediatric Endocrinology across Europe
}

\author{
Jipu Jiang, Richard Sinnott, Anthony Stell, John Watt, and Faisal Ahmed
}

\begin{abstract}
Paediatric endocrinology is a medical specialty dealing with variations of physical growth and sexual development in childhood. Genetic anomalies that can cause disorders of sexual development in children are rare. Given this, sharing and collaboration on the small number of cases that occur is needed by clinical experts in the field. The EU-funded EuroDSD project (www.eurodsd.eu) is one such collaboration involving clinical centres and clinical and genetic experts across Europe. Through the establishment of a virtual research environment (VRE) supporting sharing of data and a variety of clinical and bioinformatics analysis tools, EuroDSD aims to provide a research infrastructure for research into disorders of sex development. Security, ethics and information governance are at the heart of this infrastructure. This paper describes the infrastructure that is being built and the inherent challenges in security, availability and dependability that must be overcome for the enterprise to succeed.
\end{abstract}

Index Terms - Data-Grids, Data security, Virtual Research Environment, Paediatric Endocrinology.

\section{INTRODUCTION}

$\mathrm{D}^{\mathrm{s}}$ ISORDERS of sex development (DSD) are a set of rare to very rare disorders affecting the genito-urinary tract and, in a lot of instances, the endocrine-reproductive system. Children born with DSD may be born with ambiguous genitalia and the resulting decision-making process of sex assignment can be extremely disturbing and difficult for parents, families and health-care professionals [1].

Given the rarity of the condition, there is an associated scarcity of data on DSD. In countries the size of Scotland for example, a dozen or so cases occur per year. To help improve the understanding and provision of therapy and potential treatments or interventions associated with DSD, there is a great

Manuscript received January 5, 2009. This work is supported by the EU under the Framework 7 e-Health program.

Jipu Jiang is a research associate at the National e-Science Centre, University of Glasgow, Glasgow, UK (phone: +44 (0) 141330 8648; e-mail: j.jiang@nesc.gla.ac.uk).

Professor Richard Sinnott is the technical director of the National e-Science Centre at the University of Glasgow, Glasgow, UK (e-mail: r.sinnott@nesc.gla.ac.uk).

Anthony Stell is a research associate at the National e-Science Centre, University of Glasgow, Glasgow, UK (e-mail: a.stell@nesc.gla.ac.uk).

Dr John Watt is a research associate at the National e-Science Centre, University of Glasgow, Glasgow, UK (e-mail: j.watt@nesc.gla.ac.uk).

Doctor Faisal Ahmed is a consultant in Paediatric Endocrinology \& Bone Metabolism, Royal Hospital for Sick Children, Yorkhill, Glasgow, UK (e-mail: gcl328@clinmed.gla.ac.uk) need to increase knowledge of the genetic and biochemical profiles which characterize DSD. Different genetic anomalies can cause different manifestations of DSD and associated other clinical complications. At the heart of much research in this area is the androgen receptor which is involved in controlling gene expression for genes associated with DSD and in determining the sex phenotype. In particular much DSD research is focused around androgen receptor co-factors that modulate sex steroid action [2].

Current European research on DSD is fragmented with vertical and cross-national integration of different but complementary disciplines. The EU FP7 EuroDSD project (www.eurodsd.eu) aims to establish a level of coherence that has so far been non-existent in this field. In so doing, EuroDSD aims to facilitate the study of multiple, complementary DSD research areas in order to make a significant step forward in the understanding of DSD. The intention is to bridge the gap between a variety of disciplines including clinical medicine, biochemistry, molecular genetics and molecular biology, through cross-national integration of resources and expertise across six European countries including the UK, France, Netherlands, Germany, Italy and Sweden.

In order to achieve this level of integration, the central component of the project is the development of a Virtual Research Environment (VRE), designed to facilitate secure, flexible collaboration using state of the art technologies. This technical work is led by the National e-Science Centre (NeSC www.nesc.ac.uk) in Glasgow and is the basis for this paper.

This paper describes the challenges in implementing the VRE for the EuroDSD project. Section 2 goes into greater detail on the background clinical context. Section 3 reviews the concept of VRE. Section 4 discusses some important issues encountered during the requirements caption and system design. Section 5 discusses the detailed infrastructure requirements of the VRE in computational terms. Section 6 describes the implementation progress and results to date and section 7 draws conclusions and identifies areas of future work.

\section{PAEDIATRIC ENDOCRINOLOGY}

The clinical motivation for EuroDSD is to improve the understanding and therefore the provision of therapy for individuals with DSD. To that end, the project has identified three key areas of research that stand to benefit greatly from the use of collaborative informatics tools such as the VRE. These 
are analyses of the genetic profiles involved in DSD; the associated biochemical processes involved, and the process of androgen action to control gene expression.

The overall project structure has been divided into six work-packages (WPs). WP1 involves the development of the VRE and WP6 focuses upon the development of a training portal for general clinical training and education on DSD. The following research areas make up the other four packages.

\section{A. Defining the Genetic Basis of DSD}

Work package WP2 aims to define and understand the genetic basis of DSD. Using state of the art re-sequencing technology, a comprehensive nucleotide sequence of the genes known to be involved in genitourinary tract development will be screened. Building on work that suggests that patients with gonadal anomalies are due to changes in gene copy number or gene microdeletion/duplication [3]-[8], the opportunity to better understand the genetic pathways involved in human gonadal development now exists. At the time of writing, a core set of genes have been identified which will form the basis for targeted gene-chips for screening of DSD and therefore provide new diagnostic possibilities.

\section{B. Enhancing Knowledge of Mediators of Androgen Action}

Work packages WP3 and WP4 are designed to study the functional consequences of DSD. A key process in this regard affected by DSD is the dependence of the sex phenotype on androgen action via the androgen receptor acting as gene expression control factor. WP3 aims to characterize the relevance of androgen receptor co-factors, which are relevant to normal pubertal development. WP4 aims to identify sex-specific methylation patterns, where the inhibition of specific bases at the DNA level can become genetic traits mediated by the androgen receptor. These studies will give highly valuable information in understanding the natural course of DSD.

\section{Defining the Biochemical Basis of DSD}

Work package WP5 aims to evaluate steroid profiles in plasma and urine using gas chromatography and mass spectrometry technologies. The results of this work package will be utilized to define steroid metabolome fingerprints, which can then be linked to distinct disorders caused by mutations in steroidogenic enzymes or their co-factors [9]-[11]. WP5 is ultimately aiming at the analysis of the complete steroid metabolome in DSD patients with hitherto unknown causes of disease to discover novel steroidogenic disorders. This will also create new diagnostic algorithms and allow development of better diagnostic strategies in conjunction with genetic discovery approaches in EuroDSD.

These key areas of research cross several disciplines, and the progress of the independent packages are mutually dependent and complementary to one another. The synergies through these work packages and overarching goals of EuroDSD are facilitated through the development, use and adoption of a targeted VRE for DSD research.

\section{VIRTUAL RESEARCH ENVIRONMENT}

A Virtual Research Environment (VRE) can be regarded as a common gateway, designed to help researchers in all disciplines manage the increasingly complex range of tasks involved in carrying out their research. Ideally a VRE should provide an extensible and personalisable framework that integrates various applications, services, and resources to support particular areas of research as a Problem-Solving Environment (PSE) [12]. The major benefit of a VRE is to support collaborative activities. That is, through a VRE, researchers from different domains, from different locations and with different but complementary research interests should be able to work cooperatively exploiting targeted tools for sharing data, for knowledge sharing and exploiting common application infrastructures.

VREs are often used to provide the user front end to e-Infrastructures, e.g. those based upon Grid technology. A common requirement for e-Infrastructures is to provide seamless single sign-on and access to distributed heterogeneous resources. Many large-scale e-Infrastructures provide common security access models for example through X509-based public key infrastructures. Advances to support finer-grained access control and authorisation are needed in domains such as DSD. To support this, local sites offering resources (including data) need to be able to define and enforce their own access policies reflecting local ethics and consent of patients. These should be seamless enforced when accessing and using these resources through the VRE.

\section{DESIGN ASSUMPTIONS}

It is intended that the EuroDSD VRE will support clinical, genetic and biochemical data access and integration from six European clinical centres providing care for patients with DSD as well as supporting an extensive portfolio of targeted services/tools to support all aspects of DSD research. The VRE will provide capabilities to link clinical data resources on a case-by-case basis as well as providing a variety of DSD-targeted bioinformatics applications/services to support the scientific research.

Due to the heterogeneous nature of the data and tools in use by the various countries across Europe, seamless management of distributed data with various format and security policies and fine-grained Grid security is needed. Ultimately it is intended that each of the six centres will make available approximately 300 well-characterized patients with DSD as the basis for a continuing data assessment during the funding period, along with large amount of biomaterial resources.

The cornerstone of the VRE model is site autonomy. Each clinical site is solely responsible for deciding what data sets it can share, with which partner sites and in what context. To support this, the design of the VRE is driven by security, incorporating both the needs of the clinical community and ethical oversight required on information governance. Where feasible it is intended that the VRE supports automatic extraction of clinical data across partner sites and their sharing across EuroDSD subject to security and ethical requirements. 
The acquisition of existing clinical and other data sets into the VRE allows for rapid assessment of populations in the proposed research clusters. Availability and fault tolerance of the VRE and associated resources are essential features of the VRE and its supporting e-Infrastructure.

\section{VRE REQUIREMENTS}

Due to cultural, historical and social considerations associated with DSD, lack of information and in turn limited resultant collaborations are two of the biggest obstacles for rare disorder research and treatment. Many DSD disorders have not yet been adequately studied, e.g. their genetic-phenotypic relationship and the impact of genetic anomalies, due to limited numbers of recorded clinical cases. In creating a VRE, incorporating clinical and genetic data from 6 national centres across Europe, EuroDSD will have a variety of patients with DSD available. This will allow for much richer, targeted studies on a previously unprecedented scale to be supported. The ability to perform detailed interrogation through a VRE of recorded cases with associated clinical information will facilitate highly efficient and targeted use of novel genetic and biochemical strategies for the identification of novel disorders associated with DSD.

Based on requirements from DSD researchers, the most important resource to be established in the VRE is a centralized registry for recording detailed clinical data sets associated with DSD. The registry provides various functionality including basic querying of DSD cases; uploading of DSD cases, and edit/delete of DSD cases. Data validation and adherence to a common data model is crucial to DSD research. The existing EuroDSD requirements capture and system design exercise has taken the following issues into careful consideration.

\section{A. Data Integration}

To design a heterogeneous data infrastructure requires a common understanding of data that may exist in different data resources across partner sites. Data heterogeneity, software and data storage heterogeneity and language heterogeneity must all be addressed to tackle this problem. To address this, a core data model has been agreed between all contributors of EuroDSD.

To support this, a scoping exercise was performed at the beginning of the project to assess the support requirements of individual partner sites. As a result, the consortium agreed to use the Scottish Genital Anomaly Network (SGAN http://www.sgan.nhsscotland.com/) template as the core dataset of the registry.

The initial phase of the project was focused on aligning the separate centre data formats into an initial core data model that captures the agreed common data sets associated with DSD. However, partner sites continue to suggest refinements and secondary information to be added for DSD research. With this in mind, the VRE registry has been developed to be flexible and scalable to include further information, often specific to individual centres, into the back-end registry data repository. This flexibility is particularly important for the biochemical and genetic information held by sites required for genetic, genomic, and steroid analysis.

Furthermore, whilst some centres have defined and use their own databases and IT systems for DSD, some sites have not yet moved to completely storing patient information electronically. Excel spreadsheets are one of the more commonly used data formats for local DSD information. This brings challenges for automatic data federation.

Based on this, three entry methods are provided: manual, bulk upload, and automatic. Manual entry requires a research nurse or local clinician to enter patient DSD clinical patient data through the VRE registry interface. Strict data validation and enforcement of data entry to adhere to the common data model is supported. Bulk upload enables Excel spreadsheets comprising many DSD patients to be uploaded in built to the registry through the VRE. In this model, numerous cases are parsed and inserted into the registry. It is also the case that where feasible, customized automatic extraction of clinical data sets across partner sites will be built with appropriate ethics approval. This model has not yet been implemented however.

\section{B. Security}

Due to the highly sensitive nature of the information being shared in the EuroDSD project, the security measures to be put in place must be enforced with the utmost rigour. The challenges faced in providing access to the distributed DSD information include: authentication; authorization; auditing; privacy and identity protection; data ownership, consent and data encryption.

\section{Authentication}

The most common way of supporting VRE authentication is via usernames and passwords. However when accessing heterogeneous resources through the VRE, each resource will has its own authentication requirements, which can mean multiple usernames and password to remember - in the worst case, a username/password combination for each resource.

The Internet2 Shibboleth framework [13] solves such problems by providing a Single Sign-On (SSO) mechanism to distributed resources within a federation. Instead of users having to register and login to each site they visit, Shibboleth provides the ability for users to authenticate to their local institution, (who are best placed to authenticate individuals). Based on this, resources that exist across the federation are then able to decide whether they accept such authentication assertions from given sites.

Although Shibboleth provides an enhanced framework and has been rolled out across the UK academic environment, it currently lacks support across all EuroDSD partner centres. For none-Shibboleth enabled organizations, the National e-Science Centre at Glasgow has provided a virtual home for EuroDSD users as their home institution for the federation.

\section{Authorization}

Building upon federated authentication models, Shibboleth provides the foundation to support and integrate Role Based 
Access Control (RBAC) authorization mechanisms. To achieve this, it is necessary to assign digitally signed attribute certificates to EuroDSD participants. These are subsequently passed through Shibboleth to the VRE (seamlessly from the end user perspectives), for use by VRE to decide which resources can be accessed, to what level and to which users.

\section{Audit tracking}

Given the importance of the EuroDSD data, accountability has been identified as a key requirement of the VRE. A community-wide policy on usage and behaviour has been agreed and any operations over the data need to be recorded for future auditing. If any suspicious or malicious activity is found, the user who performed such action will be banned from the VRE to protect future patient data being tampered with.

Users are treated equally in the VRE with their Shibboleth Id used as the unique identifier for users. The access to and usage of the portal is also monitored using the Google Analytics software (www.google.com/analytics/).

\section{Privacy and identity protection}

The purpose of the registry is to help clinicians search for DSD cases within Europe and beyond. If a suitable patient is found in the registry, e.g. for a particular DSD study, the researcher or clinician can contact the owner of the record for further information. The DSD records themselves do not contain any patient identifying data themselves, but they do include information on who added the case to the registry. Indeed the information provided in the SGAN template has been carefully and deliberately screened to avoid the possibility for identifying individuals. Thus for example, the Date of Birth in the SGAN template has been registered as Year of Birth in the VRE to help minimize the chance of patient identification. The demographic component of the registry only covers information relating to the clinical centre and the clinician that has uploaded the case.

However, local identifiers, which are essential to retrieve the patient's record in the hospital, can potentially reveal the identity of the patient. Given this, the local identifier of every patient must be encrypted for everyone except the record owner in the VRE.

Further follow up through the registry, e.g. for exchange of biomaterials on particular cases is, subject to ethical approval, currently achieved through direct contact with the consulting clinician. We note also that the clinical data registry itself includes detailed information on whether such biomaterials are available and whether they have been screened for particular genes.

\section{Data ownership and consent}

Each clinical site is solely responsible for deciding what data sets it can share, with which partner sites and in what context. This brings up the issue of ownership and consent of patients records. Records in the registry are owned by the person who uploads them. Only the owner can have the right to edit and delete the records. This gives the owner the right to decide what to share and with whom.

The owner can also decide who can see the records and in what context. The level of clinical patient consent is crucial here. To share data on the registry, clinicians must obtain consent from the patient or next of kin. Different countries have different policies regarding consent. For example, in UK, unless the patient has specifically opted in to the EuroDSD study, the data can be shared within the country, i.e. the UK, but not throughout Europe. To overcome the policy differences, the consortium has agreed on four levels of consent: local, e.g. for a particular clinic only; national, e.g. for sharing in a given country only; consent for sharing across the EuroDSD collaborators, and consent for sharing across the international community incorporating wider non-EuroDSD partners. Therefore, the VRE must be able to store the consent status of each record and enforce the consent level when users search these records. For example, a case enter from UK with consent of national level can only be seen by centres and researchers registered in UK, e.g. London, Cambridge and Glasgow. Other European countries cannot see such record.

\section{Encryption}

A further challenge in security is to protect the various data-sets whilst in transit from centres to the registry from eavesdropping, interception and modification. This is achieved by securing the channels of input and data transit using SSL over HTTPS. This is achieved as part of the Shibboleth authentication mechanism.

The data at rest in the VRE repository is also similarly protected using encryption of data before it is uploaded and downloaded. This is done in an application-layer module between the VRE portal and the back-end associated database.

\section{Usability}

To minimize the learning curve of the EuroDSD VRE, usability is extremely important for the clinical experts from multiple disciplinary backgrounds. The VRE must be easy to use and information must be clearly presented. This has been taken into consideration when designing the VRE for clinicians and scientists. A simple colorful theme has been used for better presentation. The JavaScript and AJAX [14] environments allow a tailored access and usage experience for users.

User feedback has been especially valuable in improving usability of a VRE. The interface has undergone numerous usability tests of clinicians and scientists, and will continue to be refined over the coming years.

\section{IMPLEMENTATION}

The EuroDSD VRE itself has been based upon a rapid prototyping approach - exploiting a body of projects in this area at NeSC in Glasgow. Early prototypes were made available to the programme members for assessment, and improvements to the prototypes were made based on those feedbacks. The logical EuroDSD architecture depicting the various partner sites is shown in Fig. 1, in which partners sites performing different functions in the project are presented with different colors. 
Based on feedback on the initial prototype and on an increasing set of requirements captured for the EuroDSD project, it was decided that a more flexible feature-set would be necessary for the VRE. Upon investigation and testing over several technologies, the Apache Struts2 [15], Spring framework [16], and Hibernate [17] were chosen for the implementation of EuroDSD VRE.

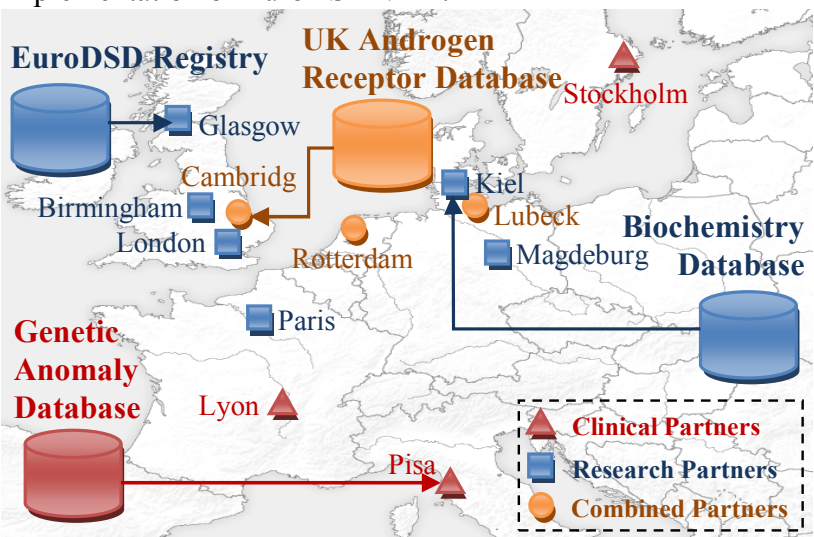

Fig. 1. Logical infrastructure supported by the VRE

To provide maximum flexibility, the VRE architecture is classified into four layers. From top to bottom, the first layer is the presentation layer that provides multiple ways to display information, e.g. HTML, JSP, AJAX, etc. Beneath this, the second layer is specially designed to control targeted workflows. The third layer provides services to process EuroDSD specific logic. And at the bottom, the fourth layer offers support for data persistence.

Based on this architecture, chosen frameworks will be deployed into the layers according to the features they provided. As a widely adopted Model View Controller (MVC) [18] framework, Struts2 will act as the controller of the VRE and provide presentation of the data. It supports the first and second layers of VRE. And Spring framework, as one of the most respected Inversion of Control (IoC) [19] container, will be in charge of the third layer to provide a wide range of services for EuroDSD business logics. Finally the Hibernate, at the bottom layer, will provide powerful yet high-performance data persistence service to the VRE.

This solution significantly improved the overall flexibility, testability, and maintainability of the VRE. It was also compatible with most existing frameworks and standards, including JSR-168 portlet. We recognise that this is extremely important for the future when integrated with existing Grid tools support genetic and biochemistry research and collaboration. In addition, more useful features offered through this architecture include better community support, support of multiple presentation layer standards, and Spring Security framework based on Aspect Oriented Programming (AOP) [20].

\section{Availability}

Most of the VRE implementation in the first prototype was rewritten for the new architecture. Thanks to the testability framework offered through Struts and Spring, the system can easily be modulated and tested. Based on this, bugs can be significantly reduced and the system robustness is increased.

Based on the four-layer architecture, business logic is properly stripped from the presentation and data. This provides better maintainability for future system administrators, who do not have to know the whole system to maintain component parts of it. Clearer separation of the business logic layer also provides flexibility in choosing service providers. Such flexibility is also useful in later integration with further resources and tools.

Further features and improvements have been implemented to provide better availability of the system. For example, it is normally the case that changes of configuration will typically require the restart of the Web server. Doing this will temporarily stop the services. However, in the EuroDSD VRE, configuration is dynamic and can be loaded into the VRE through scripts and property files. Such implementation models significantly reduce the number of server restarts and provide consistently available services to the EuroDSD community.

\section{Security}

In terms of security, the Shibboleth system is fully integrated into the VRE. Everything inside VRE must run through the Shibboleth authentication process. Users not at institutions involved in a federation have been offered a virtual home at NeSC-Glasgow. Based on consortium security requirements, each user has been assigned with roles, which are used for fine-grained access control. Two key roles in particular have currently been identified: EuroDSD_contributor and EuroDSD_researcher. A user with the EuroDSD_contributor role has the privilege to upload, edit, and withdraw cases in the registry, whilst users assigned the EuroDSD_researcher role can only search patient data.

As identified by the consortium, patient information registered in VRE must retain anonymous and untraceable to patients. Therefore, the services have been implemented to take the responsibility to provide anonymous records for none-owners. Consent level enforcement is also achieved at this layer too. Such implementation allows ensuring that no sensitive information will be passed out beyond the VRE.

So far the VRE implementation has secured every operation in the VRE (search, upload, edit, delete) according to roles assigned to individuals. As noted, HTTPS is used to encrypt communications between user browsers and the VRE, and between the VRE to data sources. Thus end users are completely oblivious of the underlying security used to enforce access and use of clinical data made available in the VRE.

\section{Usability}

Usability is extremely important for non-IT experts to use the VRE. This has been done in two key ways. Firstly, the VRE hides as much complexity as possible and provides unified access to heterogeneous resources. Secondly, the Human Computer Interface (HCI) components of the VRE have been developed to be as straightforward and easy to use as possible.

Bearing this in mind, the implementation provides a unified access interface with user driven upload and query fields 
supported. Wherever possible, selection boxes are used instead of text fields to ensure unified data formats (aligned with the core data model) and avoiding data entry confusion. For DSD it is important fill in a variety of DSD information. JavaScript has been used in the user interface to provide data validation and automatic calculations when possible. For example, the External Masculinisation Score is calculated based on user selections of five clinical fields. Invalid data and actions are shown immediately when moving to next data entry fields or on request submission. Last but not the least, AJAX technology is used to provide coherent flow of information without the need for page refreshing to interrupt user experience.

\section{Progress}

The first production system has been deployed online to provide $24 * 7$ availability to EuroDSD clinicians and research groups since 1st December 2008. Clinical cases from multiple sites have been recorded and are now being accessed in the registry. Currently, 53 patient data has been added to the registry from three centres across Europe, and this number keep growing. At the same time, further requirements will be gathered from consortium to identify necessary tools for data analysis and collaborations. These tools will be integrated seamlessly into the VRE on top of the patient data collected.

\section{CONCLUSIONS}

The work on the EuroDSD project is still very much on going. At present the focus has been very much upon support of the registry but next steps are looking more at collaborative tools to be offered through the VRE. This includes wikis discuss particular cases in the registry for example. The bioinformatics tool support and management of genetic data sets supported for analysis of novel genes will also be supported in the next version of the VRE. In this regard, bioinformatics applications supporting for example sequence analysis through BLAST [21] are currently being deployed. These will make use of large-scale HPC facilities such as ScotGrid (www.scotgrid.ac.uk) and the UK e-Science National Grid Service (www.ngs.ac.uk) - subject to ethics considerations on data use on HPC facilities.

\section{ACKNOWLEDGMENT}

The research leading to these results has received funding from the European Community's Seventh Framework Programme (FP7/2007-2013) under grant agreement number 201444. The authors would like to acknowledge the ESPE DSD consortium that has contributed to the development of the prototype registry. We would also like to acknowledge the other partners in the EuroDSD project: Prof. Olaf Hiort, Dr. Ken McElreavey, Prof. Ieuan Hughes, Prof. Wiebke Arlt, Prof. Stenvert Drop, Prof. Paul-Martin Holterhus, Dr. Ralf Werner, Dr. John Davies, Prof. Lendert Looijenga, Dr. Martine Cools, Prof. John Achermann, Prof. Silvio Bertollini, Prof. Olle Soder, Dr. Anna Nordenstrom, Prof. Peter Wieacker, Dr. Susanne Ledig, Prof. Yves Morel, Dr Ole Ammerpohl, and Dr Felix Riepe.

\section{REFERENCES}

[1] Hiort $\mathrm{O}$, Holterhus PM, Thyen U. "The basis of gender assignment in disorders of somatosexual differentiation". Horm Res 64 (Suppl.2), 2005, $18-22$.

[2] Bebermeier JH, Brooks J, DePrimo S, Werner R, Deppe U, Demeter J, Hiort O, Holterhus PM. "Cellline and tissue specific signatures of androgen receptor coregulator transcription". J Mol Med, 2006, 84: 919-31.

[3] Tar A, Solyom J, Gyorvari B, Ion A, Telvi L, Barbaux S, Souleyreau N, Vilain E, Fellous M, McElreavey K. "Testicular development in a 46,XX true hermaphrodite with a deletion of distal Xp sequences", 1995, Hum. Genet. 96: 464-468.

[4] Wieacker P, Missbach D, Jakubiczka S, Borgmann S, Albers N. "Sex reversal in a child with the karyotype 46,XY", 1996, dup (1) (p22.3p32.3). Clin Genet. 49: 271-3.

[5] Veitia RA, Nunes M, Quintana-Murci L, Rappaport R, Thibaud E, Jaubert F, Fellous M, McElreavey K, Goncalves J, Silva M, Rodrigues JC, Caspurro M, Boieiro F, Marques R, Lavinha J. "Swyer syndrome and $46, \mathrm{XY}$ partial gonadal dysgenesis associated with $9 \mathrm{p}$ deletions in the absence of monosomy-9p syndrome", 1998, Am J Hum Genet. 63: 901-5.

[6] Aleck KA, Argueso L, Stone J, Hackel JG, Erickson RP. "True hermaphroditism with partial duplication of chromosome 22 and without SRY”, 1998 Am J Med Genet. 85: 2-4.

[7] Bardoni B, Zanaria E, Guioli S, Floridia G, Worley KC, Tonini G, Ferrante E, Chiumello G, McCabe ERB, Fraccaro M, Zuffardi O, Camerino G. "A dosage sensitive locus at chromosome Xp21 is involved in male to female sex reversal", 1994, Nature Genet. 7: 497-501.

[8] Ogata T, Muroya K, Sasagawa I, Kosho T, Wakui K, Sakazume S, Ito K, Matsuo N, Ohashi H, Nagai T. "Genetic evidence for a novel gene(s) involved in urogenital development on 10q26", 2000, Kidney Int. 58: 2281-90.

[9] Arlt W, Walker EA, Draper N, Ivison HE, Ride JP, Hammer F, Chalder SM, Borucka-Mankiewicz M, Hauffa BP, Malunowicz EM, Stewart PM, Shackleton $\mathrm{CH}$. "Congenital adrenal hyperplasia caused by mutant P450 oxidoreductase and human androgen synthesis: analytical study", 1994, Lancet, 363: 2128-2135.

[10] Draper N, Walker EA, Bujalska IJ, Tomlinson JW, Chalder SM, Arlt W, Lavery GG, Bedendo O, Ray DW, Laing I, Malunowicz E, White PC, Hewison M, Mason PJ, Connell JM, Shackleton CH, Stewart PM. "Mutations in the genes encoding 11 $\beta$-hydroxysteroid dehydrogenase type 1 and hexose-6-phosphate dehydrogenase interact to cause cortisone reductase deficiency", 2003, Nature Genetics, 34: 434-439.

[11] Shackleton C, Marcos J, Malunowicz EM, Szarras-Czapnik M, Jira P, Taylor NF, Murphy N, Crushell E, Gottschalk M, Hauffa B, Cragun DL, Hopkin RJ, Adachi M, Arlt W. "Biochemical diagnosis of Antley- Bixler syndrome by steroid analysis", 2004, American Journal of Medical Genetics A, 128: 223-231.

[12] E. Gallopoulos, E. Houstis, and J.R. Rice. ProblemSolving Environments for Computational Science. IEEE Computational Science and Engineering, 1:11-23, 1994.

[13] The Internet2 Shibboleth framework http://shibboleth.internet2.edu

[14] Jesse James Garrett (2005-02-18). Ajax: A New Approach to Web Applications. AdaptivePath.com. Retrieved on 2008-06-19. Available: http://www.adaptivepath.com/ideas/essays/archives/000385.php

[15] Struts2 Framework. Available: http://struts.apache.org/2.x/

[16] Spring Framework. Available: http://www.springsource.org/

[17] Hibernate. Available: http://www.hibernate.org/

[18] Trygve M. H. Reenskaug (1978). MVC. XEROX PARC 1978-79. Available: http://heim.ifi.uio.no/ trygver/themes/mvc/mvc-index.html

[19] Martin Fowler (2004). Inversion of Control Containers and the Dependency Injection pattern. Available: http://martinfowler.com/articles/injection.html\#InversionOfControl

[20] Kiczales, Gregor; John Lamping, Anurag Mendhekar, Chris Maeda, Cristina Lopes, Jean-Marc Loingtier, and John Irwin. "Aspect-Oriented Programming", 1997, Proceedings of the European Conference on Object-Oriented Programming vol.1241. pp. 220-242.

[21] Sinnott,R.O. Stell,A.J. Bayer,M.M. Koetsier,J. "Grid Infrastructures for Secure Access to and Use of Bioinformatics Data: Experiences from the BRIDGES Project". The First International Conference on Availability, Reliability and Security (ARES-2006), Vienna, Austria, April, 2006. 


\section{University Library}

\section{- M M N E R VA A gateway to Melbourne's research publications}

Minerva Access is the Institutional Repository of The University of Melbourne

\section{Author/s:}

Jiang, J;Sinnott, R;Stell, A;Watt, J;Ahmed, F

Title:

Towards a Virtual Research Environment for Paediatric Endocrinology across Europe

Date:

2009-01-01

Citation:

Jiang, J., Sinnott, R., Stell, A., Watt, J. \& Ahmed, F. (2009). Towards a Virtual Research Environment for Paediatric Endocrinology across Europe. Capoello, F (Ed.) Wang, CL (Ed.) Buyya, R (Ed.) CCGRID: 2009 9TH IEEE INTERNATIONAL SYMPOSIUM ON CLUSTER COMPUTING AND THE GRID, pp.496-+. IEEE. https://doi.org/10.1109/CCGRID.2009.68.

Publication Status:

Published

Persistent Link:

http://hdl.handle.net/11343/28784 